\title{
Management of warfarin in atrial fibrillation: views of health professionals, older patients and their carers
}

\author{
Beata V Bajorek, Susan J Ogle, Margaret J Duguid, Gillian M Shenfield and Ines Krass
}

$\mathrm{D}$ espite evidence to support its use in atrial fibrillation, ${ }^{1,2}$ several Australian studies have shown that warfarin therapy remains underutilised, particularly in older patients, ${ }^{3,4}$ where the day-to-day management is perceived by clinicians to be more difficult. ${ }^{5,6}$ In prescribing warfarin, current practice often involves initiation of therapy in the acute care setting (hospital), with long-term management provided in the community by the general practitioner. For the patient, this usually translates to routine visits to the pathology service for blood tests, regular follow-up by doctors for warfarin dose adjustment, and lifestyle changes (eg, diet).

Previous research has focused on identifying the barriers to warfarin use and preferences for treatment options. ${ }^{5,7,8}$ Widespread consultation with key prescribers and patients on how to improve warfarin use and its day-to-day management is lacking. Furthermore, to our knowledge, no studies have explored the broader perspectives of patients' carers or other health professionals. We aimed to explore the attitudes of doctors, other health professionals, patients and carers, to identify ways to improve the management of warfarin in older patients.

\section{METHODS}

A series of group interviews, involving health professionals, patients and carers, was conducted at Royal North Shore Hospital, Sydney between 1 March and 30 April 2003. Each interview involved an independent facilitator experienced in conducting focus groups and unknown to the participants, a co-facilitator, and a scribe. Discussion was facilitated using a brief topic guide, listing open-ended questions such as:

- What has been your experience with warfarin therapy?

- How do you feel about taking/managing warfarin?

- How could warfarin use be improved?

\section{Participants}

Participants were recruited using a hybrid of criterion-based sampling (ie, selecting people involved with warfarin), with maximum variation, ${ }^{9}$ providing externally homogenous (eg, comprising patients, hospital clini-

\section{ABSTRACT}

Objective: To identify the views of health professionals, patients and their carers on strategies to improve the use and management of warfarin in older patients with atrial fibrillation.

Design: Qualitative study based on analysis of group interviews.

Setting: A major metropolitan teaching hospital, from 1 March to 30 April 2003.

Participants: 14 patients ( $\geqslant 65$ years) with established atrial fibrillation and taking warfarin, three carers, 12 specialists, eight general practitioners, six community pharmacists, nine hospital pharmacists, and 11 nurses volunteered in response to flyers promoting the study.

Results: Suggested strategies to improve warfarin management targeted support services for GPs and patients. Hospital-based clinicians felt that dissemination of trial evidence to GPs to support treatment recommendations is required, and that GPs need to enlist allied health professionals in the management of patients taking warfarin. GPs preferred access to practical advice from expert colleagues on the day-to-day management. Patients requested more information about warfarin therapy, as access to information is inadequate, particularly from primary sources (GPs, community pharmacists). Verbal and written information are equally important, but a single counselling session or supply of a booklet was viewed as inadequate. Participants identified various interventions for all levels of warfarin management; from the collective input, a framework for management strategies was developed.

Conclusions: Health professionals and patients require more customised information to support warfarin use and management.

MJA 2007; 186: 175-180

See also page 197

cians, GPs, nurses or pharmacists) but internally heterogeneous (ie, differing in sex, culture and experience) groups. ${ }^{10}$

Patients were recruited by voluntary response to flyers within the hospital and the local community (aged care centres, hostels and retirement facilities). For recruiting health professionals, flyers were distributed to relevant hospital departments through the mail system and at case conferences, clinical seminars and journal clubs. Flyers were emailed or faxed to GPs listed with the Northern Sydney Division of General Practice, and to community-based nurses and pharmacists listed on an aged care services directory.

All participants were offered a nominal payment of $\$ 25.00$ plus reimbursement of travel expenses, and refreshments, once they gave informed written consent. Northern Sydney Health Ethics Committee approved the study.

\section{Data analysis}

The audiotaped discussions were transcribed verbatim then thematically analysed, using a phenomenological approach,,${ }^{10,11}$ by inductively coding significant statements within the transcripts into themes, followed by re-review of the data for verification. ${ }^{12}$ Validity was attained by investigator triangulation and respondent validation; the cofacilitator and scribe independently analysed the transcripts before reaching a consensus, ${ }^{13,14}$ then compared this against the scribe's supplementary notes. Finally, after review by the main facilitator, the findings were fed back to volunteer participants from each group to ensure the accuracy of the interpretation. ${ }^{14}$

\section{RESULTS}

From eight group interviews ( $n=63$, Box 1$)$, four core themes arose: day-to-day experiences with warfarin therapy; dependence on the GP in warfarin therapy; barriers to warfarin use; and strategies to improve warfarin use. The last theme is the subject of this article, and comprised four sub-themes:

- need for information to support warfarin use; 


\section{Characteristics of the participants in the eight focus groups}

\section{Number (Group composition)}

Patients and carers I

7 (6 patients, 1 carer; 3 M, 4 F)

Patients and carers II

10 (8 patients, 2 carers; 5 M, 5 F)

Clinicians I: aged care

6 (5 consultants, 1 registrar; 2 M, 4 F)

Clinicians II: cardiology/general medicine

6 (3 consultants, 3 registrars; 4 M, 2 F)

\section{General practitioners}

8 (3 from small practices of $\leqslant 2$ GPs, 5 from large practices of $>2 \mathrm{GPs} /$ medical centre; $6 \mathrm{M}, 2 \mathrm{~F}$ )

\section{Aged care nurses}

11 (3 aged care ward nurses, 2 aged care clinical nurse specialists/consultants, 4 home-nursing service or assessment nurse, 1 hospital-tocommunity liaison nurse, 1 hospital-to-community liaison pharmacist; $0 \mathrm{M}, 11 \mathrm{~F}$ )

\section{Hospital pharmacists}

9 (4 specialist pharmacists [cardiology, aged care, neurology], 4 rotational/ward pharmacists,

1 outpatient pharmacist; $1 \mathrm{M}, 8 \mathrm{~F}$ )

\section{Community pharmacists}

6 (5 senior community pharmacists, 1 graduate pharmacist; $5 \mathrm{M}, 1 \mathrm{~F}$ )

* Years with warfarin means number of years practising in specialty, or number of years taking warfarin, or number of years caring for a warfarin user, for health care professionals, patients, and carers, respectively. $\mathrm{M}=$ male. $\mathrm{F}=$ female. Each group interview lasted an average of 1 hour (range, 45-80 minutes).

- deficiencies in the provision of information to patients;

- access to dedicated services in the community setting; and

- delegation of responsibility for warfarin therapy.

\section{Need for information to support warfarin use}

Across all of the groups, an overarching theme was the perceived paucity of information to support warfarin therapy across the continuum of care.

GPs and hospital-based doctors felt that there is insufficient information to support the decision to initiate warfarin therapy. GPs were perplexed about the decisions made in hospital to begin warfarin therapy, and wanted a wider dissemination of clinical trial evidence to provide a rationale. They requested that specialists be more proactive

\section{Information for decision making}

$78.9 \pm 4.1(72-83) \quad 11.0 \pm 12.4(1-29)$

$76.0 \pm 9.2(65-91) \quad 3.1 \pm 4.6(0.5-15)$

$42.0 \pm 10.7(32-57) \quad 10.75 \pm 10.1(3-26)$

$41.0 \pm 13.8(28-57) \quad 12.0 \pm 10.7(2-25)$

$51.8 \pm 11.1(42-75) \quad 20.9 \pm 12.9(10-49)$

$42.5 \pm 10.4(25-54) \quad 18.9 \pm 11.1(3.5-33)$

$39.6 \pm 11.8(26-56) \quad 17.9 \pm 13.2(3-36)$

$50.5 \pm 16.9(23-67) \quad 24.9 \pm 17.3(0.5-40)$

\section{Mean age in Mean years with years \pm SD (range) warfarin ${ }^{\star} \pm S D$ (range)}

uncertainty as to whether patients' cognitive, functional and social status are considered in these decisions. Geriatricians focused on the risks of therapy, asking prescribers to justify their decisions, and cardiologists felt that GPs particularly need to engage in this process, recommending specific "prompts" (decision aids) to help assess and document a patient's suitability for warfarin therapy. Hospital pharmacists suggested explicit, objective and practical measures to routinely obtain patient-specific information for decision making, as a riskreduction strategy.

We did a [mini mental state examination] on everyone ... [I] raised the goalpost to make it that [patients scoring] above 22 out of 30 could self-medicate and were cognitively good enough to be able to do it. [Hospital pharmacist]

\section{Information for ongoing management}

To support ongoing management in the community, there was widespread need for more information, particularly among GPs, who wanted practical and interactive advice, rather than position statements, for dealing with acute situations (eg, high international normalised ratios [INRs], bleeds).

Everybody is different ... a $40 \mathrm{~kg} 90$ year-old lady is going to be different to a 30-year-old fat guy ... you can't have exactly the same for everyone, but I have never heard a haematologist talk about how warfarinisation should be done. [GP]

A warfarin update session would be really useful ... because we are out there ... day to day ... prescribing it and testing it. [GP]

Cardiologists and hospital pharmacists supported this, but varied in their approaches. Cardiologists focused on practical, simply formatted, written information for managing day-to-day situations, as continuing education seminars were of limited benefit, whereas pharmacists felt that written methods would be ineffective.

[An] educational device for the GPs ... a card that you either stick on the wall or put in your top drawer ... a quick glance ... how to start them off on warfarin, do the INRs, when to make an adjustment. [Cardiologist]

Perhaps an article [in a medical journal] as GPs have to do obligatory [continuing medical education] but quite often you reach the doctors who are pretty 
well informed anyway. [Hospital pharmacist]

Among all of the health professionals, community pharmacists felt that they have least opportunity to assist patients and GPs, as they often lack access to both specific information on the patient's warfarin regimen and appropriate resources, and are unable to track blood test results because they are not linked into the reporting system between the patient, pathologist and GP.

You make an effort to keep a record... of what they are taking ... [and] ask them each time, but if you print it on the bottle it is likely to change [to a] different dose altogether. It could change three times. [Community pharmacist]

Hospital-based pharmacists acknowledged this problem, and suggested that community pharmacists could make better use of their computer software to help manage patients.

\section{Deficiencies in the provision of information to patients}

The participants universally agreed that the provision of information to patients is inadequate, including poor use of existing resources. The supply of the standard "little blue warfarin booklet" (manufacturer-produced) is insufficient as a stand-alone measure, as patients need to practically engage with its contents to achieve adherence. Patients and carers felt that verbal and written information are equally important, but need to be provided in a paced manner with an opportunity to verify the patient's understanding at a later time.

Yesterday ... another young person got a [deep vein thrombosis] and was put on warfarin ... she went [to the community pharmacy] with her prescription, waiting for the pharmacist to counsel her and got absolutely zip counselling. So after she got the tablets she asked, "Oh, actually, have you got a book? I would like a book". "Oh no, no, I don't think so." She was shocked. [Hospital pharmacist]

Better use of the books... if they had the current dose written and they brought that with them... it could be checked by the pharmacist and GP. [Community pharmacist]

I would personally prefer that I read the book first and then come back and say
"I am not sure about this or that; could you explain this to me?" [Patient]

The cardiologist ... says you should cut out certain food, vegetables, or reduce the quantities... I found it very difficult to sit on the other side of his desk and try and make notes of what he was saying. Now to him it is a regular thing, it is a routine thing ... for me it is the first time I hear this. For 50 years I have been eating this or that... I don't think it should be left verbally ... [You] should be given a briefing sheet or something to take away ... [then] see your GP and he follows this up with verbal advice. [Patient]

\section{Need for tailored information}

Both health professionals and patients felt that existing written information is suboptimal in content, with regard to the day-today warfarin management issues that appear to cause most problems, including drug interactions, complementary medicines, and dietary vitamin $\mathrm{K}$ intake. A suggested strategy for conveying this information was "take-home" information tailored for older patients.

An anticoagulant card... lists all the interacting medications and things like that ... a list of things they could take and couldn't take and what they couldn't eat, and it's in big writing in a bigger book and easier to read. [Cardiologist]

Patients wanted detailed information to increase their confidence in the therapy, including better explanations of the reasons for taking warfarin, how it works, how dose adjustments are made, and observed phenomena (eg, bruising, variable INR results). They expressed dissatisfaction with the lack of precautionary information about drug interactions, as verbal advice was sometimes retrospective.

I certainly get interactions with different things, but I haven't been told ... [I took] antibiotics for this bad flu that I had... no, no I hadn't [been warned beforehand] and I was a bit surprised, but once you get the bad [INR] reading then [the doctor] says, "Oh yeah that was caused by such and such". But you already knew that. [Patient]

\section{Alternative modes of education}

A need to provide broader information about warfarin, to raise its profile and dispel some of the myths, via other modalities and enlisting other allied health carers, was highlighted. Hospital pharmacists favoured a television campaign, given its effectiveness in other areas of medicine and the opportunities to use an array of health and medicine-based television programs (eg, All Saints, Good Medicine).

Education is the way to go, really. Perhaps if we put it in bigger terms ... a short documentary ... so that they are not so fearful as soon as you mention warfarin. [Hospital pharmacist]

A nurse is capable of educating a patient, but what about pathology collectors? [Hospital pharmacist]

\section{Access to dedicated services in the community setting}

\section{Patient surveillance}

Community-based surveillance and support of older patients was proposed by hospital pharmacists and nurses, who recognised that education alone would not guarantee risk-free warfarin use. Mobile communitybased services were recommended, as hospital-based anticoagulant clinics were considered of limited assistance to the at-risk older population. GPs suggested specialised community liaison services, including home visits, using qualified pharmacists to improve the surveillance of patients, but were opposed to a greater role for the general community pharmacist, as they were perceived to be underqualified to provide higher level cognitive services (eg, patient education, counselling, and therapeutic drug monitoring).

In the elderly, you are still going to have a proportion who, regardless of education and good GPs, because of what they have to do themselves, are not going to be capable... you could educate them until you are blue in the face and they still wouldn't be able to see the difference between a purple and a green tablet. If warfarin is the best thing for that selected few, then perhaps they need community help for administration. [Hospital pharmacist]

If they are in there looking at the patient in-house ... then that is probably quite useful ... I usually don't have the time ... If the pharmacist is actually sitting down with them and looking at how they are managing their tablets at home ... [monitoring] compliance [and] suggesting that the dosette is the way to go 
with this patient ... It's a very practical way actually. [GP]

\section{Access to information and resources}

There was general agreement that access to information and resources within the community setting needs improvement, as education in the hospital setting was perceived as ineffective. Even patients who received comprehensive education in hospital stated that they found it difficult to apply the knowledge once discharged, as there is no ongoing reinforcement. Face-to-face accessible support and advisory services in the community setting are necessary, but patients did not feel that GPs alone could offer this.

I was in hospital and the pharmacist came and read the book to me in the hospital. She spent a long time making me understand... I just accepted it... lots of information, as much as I needed, but when I got home and practised, I found [the INR] was up, every time I rang the doctor, every 2 weeks I would give blood and it would alter. I just wondered, what is the good of it? [Patient]

I go to an endocrinologist. There is a diabetes educator attached to the practice... who fills me in and gives me all the reasoning and good motivation to keep at it ... I wouldn't mind there being ... a warfarin blood-thinning educator around the traps that you could phone or go to. [Patient]

Although community pharmacists are ideally placed and trained to contribute to ongoing patient education, hospital pharmacists were disappointed that their community peers contributed minimally to warfarin education, but GPs were worried about increasing their involvement. Patients reported variable experiences at community pharmacies, and were most satisfied when information was provided in a caring atmosphere.

It's not like they can't educate them, but I don't think they are aware that there are people out there that don't know anything about their warfarin. [Hospital pharmacist]

If they are going to be explaining warfarin again, I would want that to be my role because I have got the responsibility if it goes wrong. [GP]

It may create conflict with the patient too ... especially if the other health professional said something [different]. [GP]

We go to [a pharmacy] just up on the corner ... they know warfarin and they always check up to see that the thing that has been prescribed is right for me to take ... a very good pharmacist... Sometimes if I get a prescription from the doctor ... she will say, I had better ring the doctor about this. [Patient]

Although community pharmacists agreed that they could be more involved in warfarin counselling, both initially and on follow-up, surveillance of patients was perceived as the role of nurses and GPs.

\section{Delegation of responsibility for warfarin therapy}

Participants assigned responsibility for managing warfarin therapy to the GP and the patient, with emphasis placed on the need for patients to assume ownership of their therapy, both in acknowledging the risks and facilitating its management, and not simply relying on the GP. However, patients felt that they already assume this role, in the absence of other support, as neither the GP nor the community pharmacist is well resourced.

"Is this new tablet alright with my warfarin?" is a valuable question that they should always ask. [Nurse]

Patients have wonderful trust in their GPs, which we don't want to interfere with, but they do seem to think that the GP is going to remember and know every detail. [Hospital pharmacist]

The local doctor is great for prescribing the next amount to take according to the INR ... but I just feel that I am a bit alone, it is a bit of a journey travelling solo. [Patient]

The doctor looked [the interaction with warfarin] up on the computer and said, "deadly, don't take any more" ... and yet I had asked the pharmacist... because [with] any new drug you like to know what it is, and she said "well there is nothing on our computer ... just watch for any black spots on you"... I have got to go back from him and feed her the information that she wasn't aware of. [Patient]

\section{Synopsis of strategies}

The strategies expressed by the participants to improve warfarin use were categorised and summarised along a continuum of processes in the use of this therapy (Box 2).

\section{DISCUSSION}

To our knowledge, this is the first study to have concurrently examined the perspectives of clinicians, allied health professionals and patients regarding appropriate strategies to improve warfarin use and management in Australia. Although studies have previously explored prescriber and patient preferences for selecting treatment options, ${ }^{5,7,8}$ or have identified interspecialty differences in prescribing, 5,15,16 none have concurrently gauged opinions on how to enhance therapeutic management.

Several important issues emerged from our study. First, despite a relative paucity of evidence about the risk versus benefit of warfarin therapy in older patients, there is room for improvement in decision-making processes, particularly in the documentation and communication of decisions. Before initiation of therapy, risk assessments need to be performed and documented more carefully, as there is uncertainty as to how rigorously clinicians consider various factors. Interventions involving comprehensive assessment, with systematic documentation and communication, can assist in selection of appropriate antithrombotic therapy for individual patients, ${ }^{17}$ and may help clarify the perceived underutilisation of warfarin in older patients

It was widely acknowledged that GPs need practical information on how to apply evidence from clinical trials to individual patients. However, while GPs preferred faceto-face contact with clinicians to discuss issues and ask questions, hospital-based clinicians and allied health professionals preferred paper-based resources. The value of live contact is highlighted by an Australian study reporting an improvement in GPs' knowledge and confidence in prescribing warfarin following face-to-face academicdetailing visits. ${ }^{18}$ Although a multitude of guidelines have been developed, ranging from small practice-based protocols to consensus statements, ${ }^{19}$ their utility is dependent on the evidence and the process by which they are developed, ${ }^{20}$ and is limited by clinicians' beliefs that guidelines are inapplicable to their patients. ${ }^{21-23}$

Previous studies examining patient preferences for using warfarin versus other therapies have not sought to identify patients' needs for resources to support the therapy. ${ }^{7}$ Patients in our study felt somewhat abandoned in their management of warfarin, and felt that they have no option other than to undertake independent research because of the limited accessibility of the GP, lack of 


\section{Potential strategies at different levels of intervention to improve long-term warfarin use and management}

Hospital-based decision-making processes for the prescribing of warfarin by clinicians

- Further clinical research to quantify the risks of warfarin in the elderly population and to identify the strict pathophysiology of stroke in the presence of atrial fibrillation

- Feedback from general practitioners about post-discharge outcomes

- Systems for the complete and official assessment of patients (eg, clinical pathways, consent forms, development of a National Warfarin Register)

Community-based decision-making processes for the prescribing of warfarin by GPs

- Wider dissemination of pertinent trial evidence to GPs, to support and promote warfarin use in this setting

- Practical consensus guidelines from clinicians to assist GPs in treatment initiation, dosage adjustment, and in the handling of difficult or adverse situations

- Refresher courses about basic warfarin pharmacology

- Explicit instructions to GPs on discharge

Long-term patient monitoring in the community setting

- Greater use of external pathology services with systems for patient follow-up for routine INR testing

- Anticoagulant clinics for centralised patient care

- Virtual anticoagulant clinics (ie, INR results, GP messages to individual patients, generic warfarin advice) that could be accessed by patients and coordinated by GPs with allied health professionals

Patient surveillance (pharmacovigilance) in the community setting

- Outreach/hospital-to-community liaison facilities to assist GPs, using allied health professionals for ongoing patient assistance with dose administration, monitoring, and pharmacovigilance

- Carer involvement (family, home-nursing services)

- Vigilance and referral by pathology services

- Devices for identifying patients (cards, bracelets)

Patient and carer education and information services

- Coordinated inpatient education, for patient and carers, with appropriate measures for verifying the patient's understanding of warfarin

- Development of appropriate counselling and educational tools for the elderly and wider community (eg, larger type for printed materials, videos, audiocassettes, television)

- Community pharmacist intervention and patient counselling

Ongoing support services for patients and carers on a long-term basis

- Readily accessible information and support services on a long-term basis, to assist patients with queries and provide consistent practical advice regarding warfarin use (eg, dietary restrictions, management of bleeds, problem avoidance)

- Patient forums to provide opportunities for voicing experiences and concerns objectively with peers

$\mathrm{INR}=$ international normalised ratio.

information from community pharmacists, and non-availability of services for ongoing support, advice, and education. These findings are concerning, given that patient knowledge regarding warfarin is generally poor, ${ }^{24,25}$ and this influences anticoagulation control. ${ }^{24}$ Yet our findings suggest there is limited effort toward rectifying this problem, perhaps reflecting an assumption by health professionals that other colleagues are educating patients. Our findings highlight that additional counselling at every oppor- tunity, from all involved, is warranted and must cater to individual patient needs rather than relying on suboptimally formatted mass-produced written information. ${ }^{26,27}$

Internationally, support services to monitor and educate patients on warfarin therapy are coordinated by allied health professionals, ${ }^{28,29}$ and in our study, participants, particularly cardiologists, felt that GPs could make greater use of allied health services (eg, pharmacists and nurses) in the community. However, community pharmacists did not see a need for their own greater involvement, and GPs perceived such services to be of limited value, preferring practical advice from consultants. The responses of both GPs and pharmacists highlight an important issue: the entire, significant responsibility for the management of warfarin therapy has been defaulted to the GP. Allied health professionals must recognise their contributions to and responsibilities in warfarin therapy, as this itself may be a major step forward in improving its management in the community setting.

\section{Study limitations}

In conducting group interviews, bias may be introduced though the facilitation of the discussions, dominance of individuals, and in the interpretation of the results. However, the main facilitator and the study investigators functioned independently, were not involved in a dependency relationship with any of the participants, and necessarily set aside all prejudgements about the topic in data analysis. ${ }^{12}$ Because recruitment was voluntary, the sample may have been biased toward those wanting to express a particular viewpoint, especially grievances, and may not be generalisable to most key players in warfarin management. However, the sample represents a diversity of experiences and perspectives on this issue.

Resource limitations precluded a larger sample size, and although each discussion was facilitated until no new issues emerged, we were unable to hold multiple sessions to ensure theme saturation, ${ }^{12}$ nor ascertain additional views (eg, junior medical officers). Because of the small number of carers, it was not fully elucidated whether their perspectives differ from those of patients; however, the perspectives of both patient and carer groups, as a whole, were similar. Because the analysis involved anonymised transcripts, we were unable to correlate perspectives with participants' age, sex, location, or therapeutic regimen.

Our study suggests that very simple measures, such as disseminating information to patients and GPs, and the participation of allied health professionals, may have some far-reaching effects in terms of improving the use and management of warfarin. Much more intervention by all health professionals is required, and further research should explore the development, implementation and evaluation of new resources and strategies, given the opportunities to adopt newer technologies. 


\section{ACKNOWLEDGEMENTS}

A grateful thank-you is extended to Dr Peter Veitch for his involvement in, and support of, this study. This project was funded by the Australian Government Department of Health and Ageing.

\section{COMPETING INTERESTS}

None identified.

\section{AUTHOR DETAILS}

Beata V Bajorek, PhD, BPharm, Lecturer in Clinical Pharmacy ${ }^{1,2}$

Susan J Ogle, MB BS, FRACP, Head of Aged Care and Rehabilitation Medicine ${ }^{3}$

Margaret J Duguid, BPharm, Director of

Pharmacy ${ }^{3}$

Gillian M Shenfield, DM, FRCP, FRACP, Head of

Clinical Pharmacology ${ }^{3}$

Ines Krass, PhD, BPharm, Associate Professor ${ }^{1}$

1 Faculty of Pharmacy, University of Sydney,

Sydney, NSW.

2 Northern Sydney Central Coast Health,

Sydney, NSW.

3 Royal North Shore Hospital, Sydney, NSW.

Correspondence: beatab@pharm.usyd.edu.au

\section{REFERENCES}

1 Atrial Fibrillation Investigators. Risk factors for stroke and efficacy of antithrombotic therapy in atrial fibrillation: analysis of pooled data from five randomized controlled trials. Arch Intern Med 1994; 154: 1449-1457.

2 Hart R, Benavente O, McBride R, et al. Antithrombotic therapy to prevent stroke in patients with atrial fibrillation: a meta-analysis. Ann Intern Med 1999; 131: 492-501.

3 Enis J. Stroke prevention in patients with nonvalvular atrial fibrillation: a current community perspective. J Clin Neurosci 1997; 4: 320-325.

4 Bajorek BV, Krass I, Ogle SJ, et al. The impact of age on antithrombotic use in elderly patients with non-valvular atrial fibrillation. Australas J Ageing 2002; 21: 36-41.
5 McCrory D, Matchar D, Samsa G, et al. Physician attitudes about anticoagulation for nonvalvular atrial fibrillation in the elderly. Arch Intern Med 1995; 155: 277-281.

6 Rodgers H, Sudlow M, Dobson R, et al. Warfarin anticoagulation in primary care: a regional survey of present practice and clinicians' views. Br J Gen Pract 1997; 47: 309-310.

7 Man-Son-Hing M, Laupacis A, O'Connor A, et al. Warfarin for atrial fibrillation: the patient's perspective. Arch Intern Med 1996; 156: 1841 1848.

8 Monette J, Gurwitz J, Rochon P, et al. Physician attitudes concerning warfarin for stroke prevention in atrial fibrillation: results of a survey of long-term care practitioners. J Am Geriatr Soc 1997; 45: 1060-1065.

9 Patton M. Qualitative research and evaluation methods. 3rd ed. Thousand Oaks, Calif: Sage Publications, 2001

10 Calder B. Focus groups and the nature of qualitative marketing research. J Mark Res 1977; 14: 353-364.

11 Wilkinson S. Focus group methodology: a review. Int J Soc Res Method 1998; 1: 181-203.

12 Creswell J. Qualitative inquiry and research design: choosing among five traditions. Thousand Oaks, Calif: Sage Publications, 1998.

13 Morgan D, Kreuger R. When to use focus groups and why. In: Morgan D, editor. Successful focus groups: advancing the state of the art. London: Sage Publications, 1993.

14 Cutcliffe J, McKenna H. Establishing the credibility of qualitative research findings: the plot thickens. J Adv Nurs 1999; 30: 374-380.

15 Lip G, Zarifis J, Watson R, et al. Physician variation in the management of patients with atrial fibrillation. Heart 1996; 75: 200-205.

16 Peterson G, Boom K, Jackson S, et al. Doctors' beliefs on the use of antithrombotic therapy in atrial fibrillation: identifying barriers to stroke prevention. Intern Med J 2002; 32: 15-23.

17 Bajorek BV, Krass I, Ogle SJ, et al. Optimizing the use of antithrombotic therapy for atrial fibrillation in older people: a pharmacist-led multidisciplinary intervention. J Am Geriatr Soc 2005; 53: 1912-1920.

18 Harper L. Warfarin treatment in atrial fibrillation. Quality Use of Medicines Mapping
Project. Canberra: Australian Government Department of Health and Ageing, 1999.

19 Hart R, Benavente O. Primary prevention of stroke in patients with atrial fibrillation. Proc $R$ Coll Physicians Edinb 1999; 29 (Suppl 6): 20-26.

20 Thomson R, McElroy H, Sudlow M. Guidelines on anticoagulant treatment in atrial fibrillation in Great Britain: variation in content and implications for treatment. BMJ 1998; 316: 509-513.

21 Chang $H$, Bell J, Devoo D, et al. Physician variation in anticoagulating patients with atrial fibrillation. Arch Intern Med 1990; 150: 83-86.

22 Bath P, Prasad A, Brown M, et al. Survey of use of anticoagulation in patients with atrial fibrillation. BMJ 1993; 307: 1045.

23 Beyth R, Antani M, Covinsky K, et al. Why isn't warfarin prescribed to patients with nonrheumatic atrial fibrillation? J Gen Intern Med 1996; 11: 721-728.

24 Tang E, Lai C, Lee $K$, et al. Relationship between patients' warfarin knowledge and anticoagulation control. Ann Pharmacother 2003; 37: 34-39.

25 Newcastle Anticoagulation Study Group. Effectiveness of anticoagulation among patients discharged from hospital on warfarin. Med J Aust 1998; 169: 243-246.

26 Horne R, Hankins M, Jenkins R. The satisfaction with information about medicines scale: a new measurement tool for audit and research. Qual Health Care 2001; 10: 135

27 Estrada C, Hryniewicz M, Higgs V, et al. Anticoagulant patient information material is written at high readability levels. Stroke 2000; 31: 29662970.

28 Fitzmaurice D, Hobbs F, Murray E. Primary care anticoagulant clinic management using computerized decision support and near patient international normalized ratio (INR) testing: routine data from a practice nurse-led clinic Fam Pract 1998; 15: 144-146.

29 Hall J, Radley A. A role for community pharmacists in the control of anticoagulant therapy. Pharm J 1994; 252: 230-232.

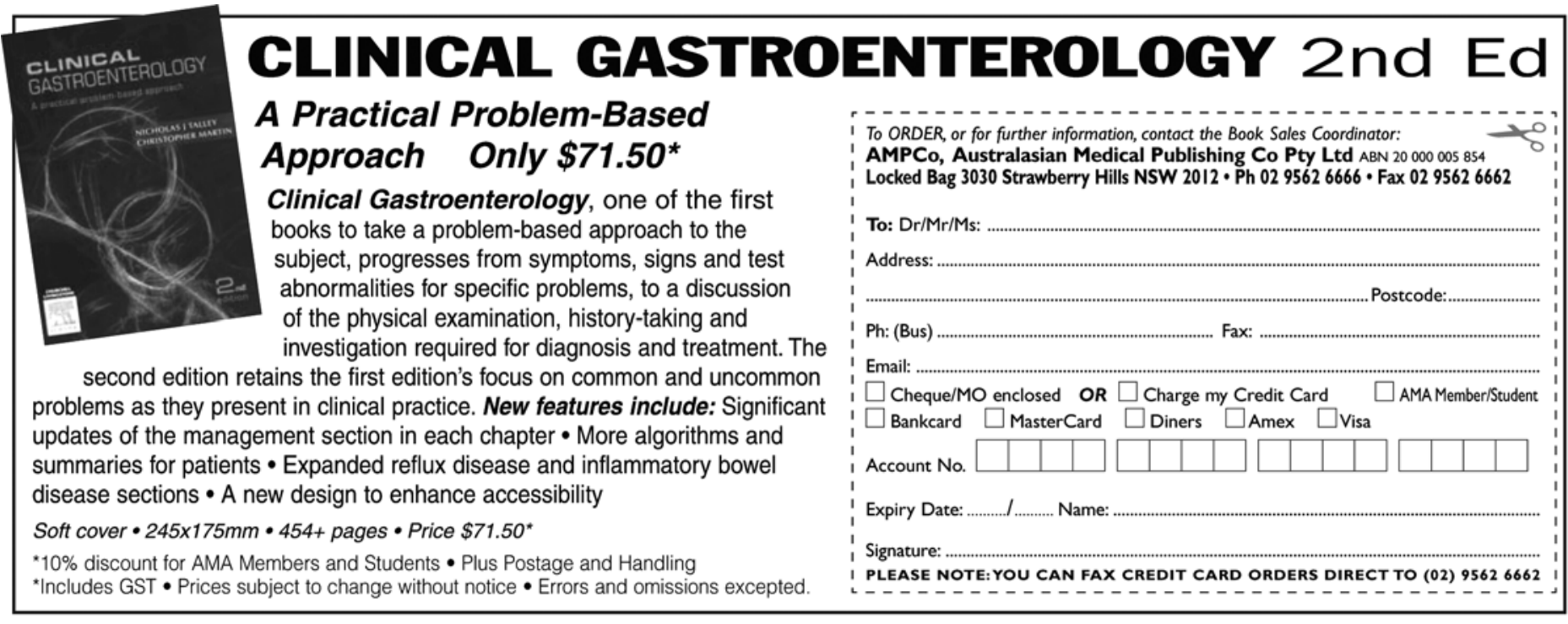

